\title{
Formation of Protease-Resistant Prion Protein in Cell-Free Systems
}

\section{Byron Caughey*}

Rocky Mountain Laboratories, National Institute of Allergy and Infectious Diseases, Hamilton, MT 59840, USA

\begin{abstract}
In transmissible spongiform encephalopathies (TSE) or prion diseases, the endogenous protease-sensitive prion protein (PrP-sen) of the host is converted to an abnormal pathogenic form that has a characteristic partial protease resistance (PrP-res). Studies with cellfree reactions indicate that the PrP-res itself can directly induce this conversion of PrP-sen. This PrPres induced conversion reaction is highly specific in ways that might account at the molecular level for TSE species barriers, polymorphism barriers, and strains. Not only has this reaction been observed using mostly purified PrP-sen and PrP-res reactants, but also in TSEinfected brain slices. The conversion mechanism appears to involve both the binding of PrP-sen to polymeric PrP-res and a conformational change that results in incorporation into the PrP-res polymer.
\end{abstract}

\section{Introduction}

The accumulation of abnormally protease-resistant prion protein (PrP-res) is common to transmissible spongiform encephalopathies (TSE). The degree of resistance of PrPres can vary depending upon the TSE strain and host species $(1,2,75)$, but the TSE-associated forms of PrPres are considerably more resistant to proteinase $\mathrm{K}$ than is the corresponding normal $\mathrm{PrP}$ isoform (PrP-sen or $\mathrm{PrP}^{\mathrm{c}}$ ). Besides having enhanced protease-resistance, the various abnormal TSE-associated forms of PrP-res (eg. PrPSc, $\mathrm{PrPCJD}^{\mathrm{C}}$ and PrPBSE) form insoluble aggregates and have ${ }^{\mathrm{B}}$ higher beta sheet content than PrP-sen $(3-6,75)$.

Many types of evidence implicate PrP-res formation as a central process in TSE pathogenesis and TSE agent replication (7-10). In the case of rare familial TSE diseases, it appears that PrP mutations can lead to aberrant behavior of PrP-sen and its spontaneous conversion to more proteaseresistant forms (see other chapters for review). However, spontaneous conversion occurs rarely, if at all, in hosts with wild type PrP-sen as evidenced by the 1 per million annual incidence of sporadic CJD in humans. Much more common in mammals is the induction of neurotoxic PrP-res formation from wild type PrP-sen upon of infection of hosts with TSE agents.

Studies with tissue culture cells and animals have revealed a considerable amount of information about the cell biology of PrP-res formation and its association with TSE pathogenesis; these subjects are reviewed elsewhere (75). Studies in cell-free systems have provided the opportunity to directly investigate PrP-res formation under much simpler and more defined conditions. Such in vitro studies have provided new insight into the mechanism of PrP-res formation and the molecular bases of TSE agent replication, strain propagation and species barrier effects. Most notably, these studies have shown that, as has long been predicted (11-15), PrP-res can itself induce the conversion of PrP-sen to PrP-res directly by a mechanism that is so specific that it could conceivably account for most of the biological manifestations of TSE agents in vivo. These cell-free studies are reviewed in this chapter.

\section{Self-Seeded PrP-res Formation}

The central proposal of most protein-only models for the infectious agent of the TSEs is that the putative infectious protein, i.e. PrP-res, directly interacts with its normal, host encoded homolog, PrP-sen, to convert it to PrP-res. In this way, it could propagate itself in the host without mediation by an agent specific nucleic acid (11-15). This fundamental ability of PrP-res to induce the conversion of PrP-sen to PrP-res (converting activity) was first demonstrated by mixing PrP-res purified from scrapie-infected brain tissue with immunoprecipitated 35S-PrP-sen and observing that 35S-PrP-sen was then transformed into 35S-PrP-res (16). This conversion was not observed in the absence of PrPres or in the presence of another type of amyloid (Alzheimer's beta). Furthermore, other labelled proteins were not converted to protease $\mathrm{K}(\mathrm{PK})$ - resistant forms in the reaction with PrP-res. Thus, the conversion reaction is PrP-specific and PrP-res dependent. The converting activity depends upon the unique conformational structure of PrP-res because, although partial, reversible unfolding of PrP-res stimulates the conversion efficiency, more complete irreversible denaturation eliminates the converting activity $(16,17)$. Further analyses of the effect of denaturants on the converting activity of PrP-res have indicated that maintenance of the native folding of a Cterminal domain ( $16 \mathrm{kDa}$ in the aglycosyl structure) is important to allow refolding and recovery of converting activity upon dilution of the denaturant (17). The denaturation of this critical C-terminal domain coincided with large reductions in both converting activity and scrapie infectivity (18).

\section{Binding vs Conversion}

The PrP-res-induced conversion of PrP-sen to the PKresistant form has now been observed in several laboratories (19-22). However, in one of the resulting papers, the authors preferred to describe it as merely a binding phenomenon rather than conversion (19). Indeed there is binding of the PrP-sen precursor to the PrP-res aggregate in the conversion process which we believe is an integral part of the conversion mechanism (see below). However, not all binding or aggregation of PrP-sen results in conversion to this PK-resistant state that is characteristic of PrP-res $(22-25,76,77)$. Although PK completely digests PrP-sen, it characteristically removes only $\sim 67$ residues 
from the N-terminus of each monomeric unit of the PrPres aggregate, resulting in 6-7 kDa downward shift in their apparent molecular weight in SDS-PAGE gels. Since nearly all of the PrP-res molecules are equivalently exposed to $\mathrm{PK}$ and similarly truncated, this type of PK resistance is not due to the nonspecific sequestration of whole PrP molecules within aggregates that are not penetrated by PK. If the latter had been the case, virtually full-length PrP molecules would have remained after PK treatment as has been observed when 35S-PrP-sen is incubated with large molar excesses of a synthetic PrP peptide fragment (19). The similar partial exposure of the N-terminal residues of both PrP-res molecules and the 35S-PrP-res products of the conversion reaction provide evidence for the incorporation of the monomers units into highly ordered polymeric structures such as amyloid fibrils. Hence, PrPsen not only binds to PrP-res, but also can convert from a PK-sensitive state to the partially PK-resistant state that is characteristic of TSE brain-derived PrP-res $(76,77)$.

Riesner and colleagues observed that acetonitrile treatment of SDS-solubilized, alpha helical and PKsensitive PrP27-30 results in aggregation of PrP and an enhancement of its total PK-resistance and beta sheet content without recovery of scrapie infectivity or fibrils (25). As was noted, this may be an example of an aggregated and PK-resistant form of PrP that is not PrPSc. On the basis of this observation, they argue that a similar apparently nonspecific aggregation process could explain our PrPres-induced conversion of 35S-PrP-sen to 35S-PrP-res. However, an important distinction must be made between their observation and ours. As described above, the 6-7 $\mathrm{kDa}$ size shift showed that the 35-PrP-res conversion products were exposed to $\mathrm{PK}$ but had the characteristic partial PK-resistance of brain-derived PrP-res (e.g., PrPSc) $(16,26)$. However, one cannot conclude the same about the acetonitrile-induced PK-resistant PrP27- 30 because there was no control in the experiments reported to indicate actual exposure of the "PK-resistant" PrP molecules to PK during the digestion procedure. Thus, the acetonitrileinduced total PK-resistance could have been due to the nonspecific isolation of PrP from PK within poorly penetrable aggregates that may be quite distinct from native TSE-associated PrP-res and the products of our cell-free conversion reaction.

As mentioned briefly above, incubation of PrP-sen with 5000-fold molar excesses of certain synthetic peptide fragments of PrP (eg., PrP90-145) can induce the formation of PK-resistant complexes that polymerize into fibrils $(19,27)$. These complexes are unstable and the associated PrP molecules do not display the same partial PKresistance of brain-derived PrP-res or the 35S-PrP-res generated in the conversion reaction induced by brainderived PrP-res. Nonetheless, these results provide another line of evidence that complex formation can lead to the formation of PK-resistant PrP polymers.

Sequence Specificity in the PrP Conversion Reaction: a Basis for TSE Species and Polymorphism Barriers? A striking aspect of the PrP conversion reaction is its sequence specificity $(20,21,28,77,78)$. Studies in transgenic mice and scrapie-infected tissue culture cells (29-31) suggested that interactions between homologous PrP-res and PrP-sen molecules were required for efficient
PrP-res formation and host susceptibility to TSE disease. In vitro studies with small synthetic PrP peptide fragments also highlighted the effects of PrP sequence mismatches in the polymerization process (32-34). More recently, the sequence specificity of interactions of the full-length PrP protein in the conversion process has been investigated directly with the cell-free system $(20,21,28,77,78)$. These studies have provided insight into molecular basis for barriers to the transmission of TSEs between species (species barriers) and same-species hosts with different PrP genotypes (polymorphism barriers).

Combinations of PrP-res and PrP-sen from several different species (mice, hamsters, sheep, cattle and human)

A. In vivo transmission

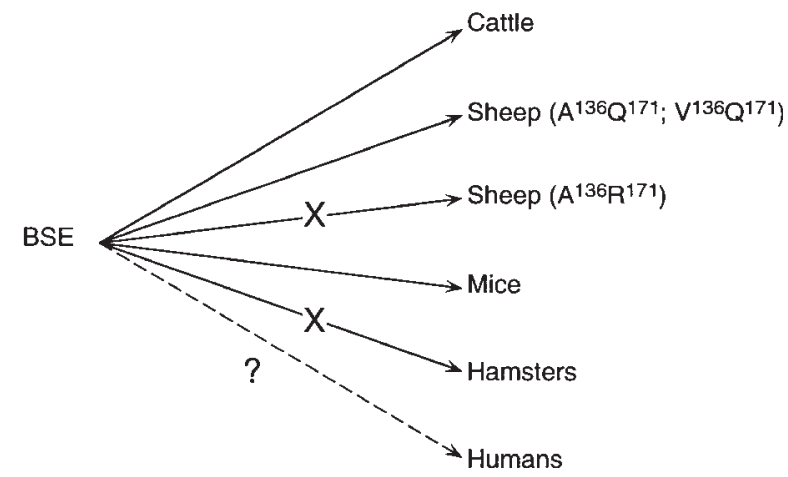

B. In vitro conversion

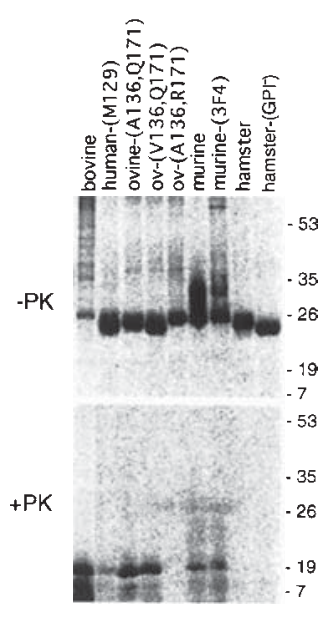

Figure 1. Correlation of in vivo transmissibilities of BSE infectivity with the in vitro conversions induced by PrPBSE. A; Known experimental or natural transmissibilities of BSE to various host species or genotypes. Although it now is apparent that BSE has transmitted to humans to give rise to new variant CJD $(73,74)$, the efficiency and extent of this transmission remains unclear. B; PrPBSE-induced in vitro conversions of different radiolabelled PrP-sen molecules as described in text and (21). Top panel shows the phosphor audioradiographic analysis of $\left[{ }^{35} \mathrm{~S}\right] \mathrm{PrP}$-sen molecules used in the conversion reaction without proteinase $\mathrm{K}$ treatment. The bottom panel shows the labelled PK-resistant $\left[{ }^{35} \mathrm{~S}\right]-\mathrm{PrP}$ products resulting from the incubation of the $\left.{ }^{35} \mathrm{~S}\right] \mathrm{PrP}$-sen molecules with (unlabelled) PrPBSE. The major [35 S]PrP-res products of interest are the expected 6-7 $\mathrm{kDa}$ lower in molecular weight than the $\left[{ }^{35} \mathrm{~S}\right] \mathrm{PrP}$-sen precursor and comigrate approximately with the $19 \mathrm{kDa}$ molecular mass marker shown on the right. Adapted from (21). 
have been tested $(20,21,28)$. To a remarkable extent, the efficiency of the cell-free conversion reactions correlated with in vivo transmissibilities of the corresponding agents within or between species. The homologous conversion reactions between PrP-res and PrP-sen molecules of the same sequence were most efficient, just as TSE agents are usually most efficiently transmitted to hosts of the same PrP genotype. In contrast, little or no conversion was observed with PrP-res-PrP-sen combinations associated with a lack of transmissibility in vivo. For example, PrPBSE readily converted bovine, murine, and two different ovine PrP-sen molecules to PrP-res and this correlates with the fact that BSE is transmissible to hosts expressing those types of PrP-sen (Fig.1). However, PrPBSE failed to convert the PrP-sen of two BSE-resistant hosts, Syrian hamsters and sheep of the $A^{136} R^{171}$ PrP genotype. Sheep PrP-res with the $V^{136} Q^{171}$ sequence efficiently converted the PrPsen of $V^{136} Q^{171}$ and $A^{136} Q^{171}$ sheep, which are susceptible to scrapie agent from $V^{136} Q^{171}$ sheep, but not the $A^{136} R^{171}$ PrP-sen of a scrapie-resistant strain of sheep (20). Results from these and other PrP-res-PrP-sen conversions have provided strong support for the concept that the sequence specificity in the conversion of PrP-sen to proteaseresistant forms modulates the interspecies or intraspecies transmissibilities of TSE agents in vivo.

Based upon the correlation between in vitro conversion efficiencies and known transmissibilities, we used the cellfree conversion system to gauge the potential transmissibility of BSE to humans (21). We found limited conversion of human PrP-sen to PrP-res driven by PrPBSE (Fig.2). Interestingly, the Met-129 human PrP-sen was more efficiently converted than the Val-129 PrP and this correlates with the fact that all of the human new variant CJD patients identified to date are homozygous for the Met-129 allele. The efficiencies of both of these heterologous bovine PrPBSE human PrP-sen conversions was much lower than those of relevant homologous conversions, yet similar to that of the conversion of human PrP-sen by sheep PrP-res. This in vitro analysis suggests
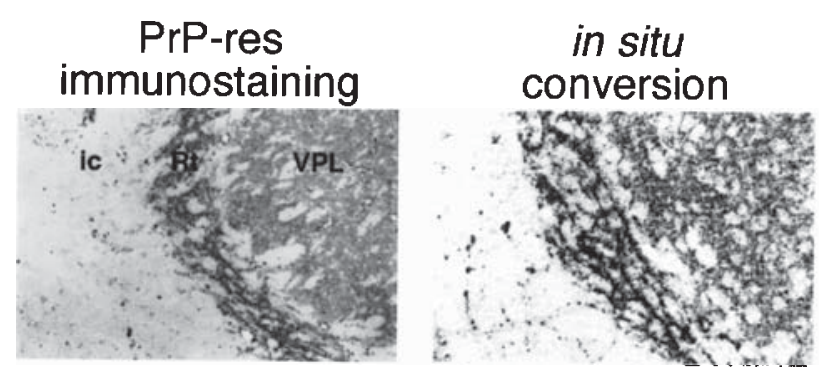

Figure 2. In situ conversion in TSE-infected brain slices. Brain slices from transmissible mink encephalopathy-infected hamsters either immunocytochemically stained for PrP-res or processed through the in situ conversion procedure(24). For the latter procedure, the slice was incubated with hamster $\left.{ }^{35} \mathrm{~S}\right] \mathrm{PrP}$-sen, treated with $\mathrm{PK}$ to remove any labelled $\mathrm{PrP}$ that had not converted to a $\mathrm{PK}$ resistant form and then dipped in photographic emulsion to expose sites of $\left.{ }^{35} \mathrm{~S}\right] \mathrm{PrP}$-res deposition. A close correspondence was observed between the patterns of staining pre-existing PrP-res and the in situ conversion product, providing evidence that the PrP-res in the slice causes the conversion of [ $\left.{ }^{35} \mathrm{~S}\right] \mathrm{PrP}$-sen to $\left[{ }^{35} \mathrm{~S}\right] \mathrm{PrP}$-res in situ. Regions shown: ic, internal capsule; Rt, reticular thalamic nucleus; VPL, ventral postlateral thalamic nucleus. Adapted from (24). that the inherent ability of these infectious agents of BSE and scrapie to affect humans following equivalent exposure may be finite but similarly low.

\section{Self-Propagating PrP-res Conformations as a Possible Basis of TSE Strains}

Strains of TSE agents can be distinguished on the basis of species tropism, incubation period, clinical disease, neuropathological manifestations and PrP-res distribution in brain tissue [for review (35)]. Numerous TSE strains have been documented even within isogenic hosts. This fact poses an interesting challenge for the protein-only hypothesis for the infectious agent: It requires that the "inheritance" or propagation of the agent strain dif ferences must be mediated by stable variations in PrP-res structure rather than mutations in an agent-specific nucleic acid. Structural differences in PrP-res have been correlated with different strains of TSEs $(2,36-38,79,80,81)$. Particularly notable are the different forms of PrP-res associated with the hyper (HY) and drowsy (DY) strains of hamster-adapted transmissible mink encephalopathy (TME). Although these PrP-res forms are both derived from Syrian hamster PrP, they are cleaved differently by PK. This suggests that they differ in conformation rather than covalent structure (2) and this has been confirmed by FTIR analysis (79). Furthermore, when incubated with hamster PrP-sen molecules, HY and DY PrP-res faithfully induce the formation of strain-specific PrP-res conversion products and thereby propagate themselves by a direct, nongenetic mechanism (26). These data provided the first direct evidence that strain-specific PrP-res polymers with the same amino acid sequence but different 3-D structures or conformations are capable of self-propagation. This is consistent with the possibility that the self-propagation of PrP-res polymers is a molecular basis for scrapie strains. Also consistent with this notion is a recent study showing that the passage of agent derived from different types of familial CJD into mice caused the accumulation of PrP-res with apparently distinct conformations (39).

\section{Correlating Scrapie Infectivity With Converting Activity,} Protease-Resistance and Aggregation of PrP-res

Given that PrP-res might be the TSE agent which depends on the converting activity for its propagation in the host, we tested whether the effects of $\mathrm{GdnHCl}$ on the converting activity, PK-resistance and aggregation of PrPSc might coincide with effects on scrapie infectivity (18). Large $\mathrm{GdnHCl}$-induced reductions in infectivity were associated with the irreversible elimination of both the proteinase Kresistance and apparent self-propagating converting activity of PrPSc. In intermediate $\mathrm{GdnHCl}$ concentrations that stimulate converting activity and partially disaggregate $\mathrm{PrP}^{\mathrm{Sc}}$, both scrapie infectivity and converting activity were associated with residual partially protease-resistant multimers of $\mathrm{PrPSc}$. These results are consistent with scrapie infectivity being related to converting activity.

In situ PrP Conversion in TSE-Infected Brain Tissue After observing the PrP-sen to PrP-res conversion reaction in a cell-free system using largely purified and concentrated reactants, efforts were made to recapitulate the conversion under conditions more like those of intact brain tissue. This resulted in the development of the in situ conversion assay (24). Infected brain tissue slices mounted on slides were 
incubated with radiolabelled PrP-sen and then treated with PK to digest any PrP-sen that was not converted to the PK-resistant form. Biochemical analysis of the tissue indicated that the expected labelled PrP-res conversion products were formed in, and bound to, infected, but not uninfected brain slices. Autoradiographic analysis of the pattern of newly formed PrP-res in situ conversion product in the infected brain slice closely matched the distribution of the pre-existing PrP-res. Punctate in situ PrP conversion was observed in brain regions containing PrP-res amyloid plaques and a more dispersed conversion product was detected in areas containing diffuse PrP-res deposits. These studies provided direct evidence that PrP-res formation involves the incorporation of soluble PrP-sen into both amyloid plaque and sub-plaque PrP-res deposits in TSE-infected brain tissue.

\section{Mechanism of PrP-res Formation: Seeded Polymerization vs Heterodimer}

The first visualizations and biochemical characterizations of PrP-res polymers (scrapie associated fibrils or prion rods) were reminiscent of amyloid fibrils (40-42). Amyloids can be composed of a number of proteins, depending upon the disease (43). Amyloid deposits are composed of linear fibrils that result from the polymerization of a usually soluble precursor protein or peptide. Seeding solutions of the precursor with pre-existing amyloid fibril fragments can greatly accelerate the polymerization of amyloidogenic proteins $(13,15)$. Amyloid polymerization often involves an increase in the beta sheet content of the constituent protein. The similarities between PrP-res and other amyloids suggested that the mechanism of PrP-res formation is like that of other amyloids $(13,15,44)$. Early support for this idea came from observations that that small synthetic peptide fragments of the PrP sequence can form amyloid fibrils $(32,33,45-48)$ and that this occurs by a seeded polymerization mechanism (32).

Recent studies with the full-length PrP-res protein have provided evidence that only ordered multimers of PrP-res, albeit widely variable in size, can induce the conversion of PrP-sen to the protease-resistant form in the cell-free system (49). The polymerized state of PrP-res also correlates with its PK-resistance, its ability to renature to full proteinase $\mathrm{K}$ resistance after partial denaturation, and with the presence of scrapie infectivity $(17,18)$. Furthermore, the in situ conversion reaction in brain slices shows that the conversion product is bound to those deposits and not released into the medium (24). These observations are also consistent with a seeded polymerization mechanism for PrP-res formation.

Because not all deposits of PrP-res in vivo show birefringent staining with Congo red or have readily visible amyloid fibril structures by electron microscopy [e.g. (50)], some investigators have argued that polymerization/ aggregation of PrP is not required for PrP-res formation $(51,52)$. For instance, the heterodimer model posits that a PrP-res exists as a monomer and that the PrP-res monomer binds to a monomer of PrP-sen to form a heterodimer $(11,14,51)$. The PrP-sen in the heterodimer then spontaneously converts to PrP-res, making a homodimer which splits into two PrP-res monomers. The fact that no proteinase K-resistant and/or high beta sheet monomer of PrP has been identified is inconsistent with this model. There have been reports of scrapie infectivity that cofractionates with monomeric forms of $\operatorname{PrP}(53,54)$, but these studies have not been confirmed $(55,56)$. Studies showing that ordered aggregates of PrP-res are active in converting PrP-sen to PrP-res demonstrate that there is, at least, no obligate requirement for a free PrP-res monomer, if one should exist, in the conversion mechanism (49).

The lack of visible fibrils in some tissue and membrane fractions containing PrP-res might readily be explained by a prevalence of short PrP-res polymers or the association of the PrP-res polymer with PrP-sen or other factors that obscure its ultrastructure and affect its birefringent staining with Congo red. In addition, there may often be small ordered, but ultrastructurally amorphous, subfibrillar structures or protofilaments which, under certain circumstances, fuse into, or seed the formation of, fibrils and amyloid plaques. Similar structures have been reported as early diabetes-associated deposits of islet amyloid polypeptide in vivo (57) and as intermediates or initiation sites for amyloid formation by synthetic Alzheimer's beta peptide (58) and PrP peptide fragments (59). Immunoelectron microscopy studies of PrP accumulation in scrapie-infected brain tissue have provided evidence for the accumulation of PrP in apparently amorphous subfibrillar forms prior to obvious amyloid fibril formation (60-62). Both the diffuse deposits and amyloid plaque of PrP-res are capable of inducing PrP conversion as shown with the in situ conversion reaction in brain slices (24).

The nucleated polymerization model is consistent with all of these observations because it predicts that PrP-res polymers ranging in size from huge amyloid plaques down to stable oligomers containing only several PrP monomers could seed the polymerization reaction. Theoretical consideration of the likely volume of a PrP monomer compared to the dimensions of classic scrapie associated fibrils suggests that fibrils containing $60 \mathrm{PrP}$ molecules might be no longer than they are wide and, therefore, would not be visible as fibrils ultrastructurally. Furthermore, unless the PrP-res polymers are long and aligned in large oriented bundles or radiating amyloid plaques that are visible by light microscopy (> 0.5 mm), staining by Congo red would not appear birefringent under polarized light. Thus, the absence of readily visible, congophilic fibrils containing hundreds or thousands of PrP-res molecules is not persuasive evidence that PrP-res is usually monomeric.

\section{The Role of Conformational Change in PrP-res Formation}

Since the available evidence suggests that there is a difference in conformation, as well as aggregation state, between PrP-res and PrP-sen (3-5), it is important to consider whether the conformational change and polymerization occur simultaneously or separately and, if separately, which event is the rate determining step in the conversion process? One possibility is that monomeric $\mathrm{PrP}(-$-sen) rapidly interchanges between high alpha helical and high beta sheet conformations on a rapid time scale (but presumably with a strong bias toward the high alpha helical conformation) and that the PrP-res-induced polymerization traps PrP in the high beta sheet conformation. In this case, the polymerization would be the rate determining step that stabilizes the high beta sheet conformer to allow the accumulation of PrP-res. A second possibility (heterodimer model) is that 
the conformational change in nonpolymerized PrP-sen is the rate-determining step which is catalyzed by nonpolymeric PrP of the high beta conformation (if it exists) and that polymerization may occur subsequently with little consequence to the rate of PrP-res formation and accumulation. A third possibility is that the conformational change and polymerization occur together and both contribute to the rate determining step. Finally, it is possible that PrP-sen molecules may first bind to PrP-res polymers in the high alpha helix conformation (or an intermediate conformation) and then more slowly convert to the high beta sheet conformation to extend the PrP-res polymer. As noted above, current evidence favors a important role for nucleated polymerization in the rate determining step of PrP-res formation and would argue against the second (heterodimer) model. Consistent with the fourth possibility are recent experiments with the cell-free conversion system which provide evidence that the binding of PrP-sen to PrP-res polymers precedes its conversion to PrP-res $(22,24,76,77)$. Otherwise, it remains to be determined which of these scenarios most accurately describes the mechanism of PrP-res-induced conversion of wild type PrP-sen to PrP-res.

\section{Potential Cofactors}

The conversion of PrP-sen to PrP-res can be induced by mostly purified, PK-treated PrP-res in the cell-free conversion reaction $(26,49)$, suggesting that these PrP reactants are all that is required under certain conditions. However, is is possible that under other conditions, such as in vivo, other factors are required for efficient conversion to occur. For instance, the binding of glycosaminoglycans to both PrP-sen and PrP-res and the potent inhibition of PrP-res formation by these same compounds and their analogs suggests that they may play an important role in vivo (63-66).

The likelihood that refolding of PrP-sen occurs during conversion has lead to suggestions that chaparone proteins might be involved (67-70) as been documented for yeast prion phenomena (71). As noted above, the PrP conversion reaction is much more efficient if the PrP-res seed is pretreated with a mild denaturant that partially and reversibly unfolds the polypeptide chain. Recent experiments have shown that certain chaparone proteins can substitute for this partial denaturation step to promote the PrP-res-induced conversion reaction (22). On the other hand, none of the chaperone proteins tested induced the conversion of PrP-sen to PrP-res in the absence of a PrPres seed. These results emphasize the importance of seeding by PrP-res and are consistent with the idea that PrP-sen is refolded during the conversion process. The stimulatory activity of certain chaparone proteins in vitro suggests that chaperones may be cofactors in the PrP conversion process in vivo.

\section{Concluding Remarks}

At this point, one might reasonably ask several important questions: Is the acquisition of protease-resistance in $\operatorname{PrP}$ due to the conformational change, polymerization, or both? And which attribute of the abnormal PrP is most important in TSE pathogenesis and transmission? Is PrP-res itself the transmissible agent? Are the most pathogenic and neurotoxic forms of PrP necessarily the most transmissible (assuming for the moment that any form of PrP is by itself transmissible)? Finally, is the sequence of events and rate limiting steps in the presumably spontaneous PrP-res formation from mutant PrP-sen in familial TSE diseases necessarily the same as the induced formation of wild type PrP-res following TSE infection?

The ultimate answers to these questions are not yet clear, but several relevant points can be made. Although the characteristic partial PK-resistance of PrP-res appears to faithfully reflect a specific abnormal conformational and/ or aggregation state that differs from PrP-sen in most cases, this characteristic is not an equally hard and fast attribute of all disease-associated forms of PrP. Because there is no $\mathrm{PK}$ in mammals, there is no reason to expect that all types of pathogenic PrP need to be resistant to PK specifically to cause disease. However, some sort of general resistance to proteolysis would likely promote both the survival of PrP-res as a potential transmissible agent and its accumulation as a pathogenic substance in the host. Nonetheless, even overexpression of wild type PrP-sen can cause neurological disease (72), so pathological (but not necessarily transmissible) accumulations of PrP can be achieved without PK-resistance under some circumstances. Since no one has clearly documented the existence of a monomeric form of $\operatorname{PrP}$ that is high in beta sheet and PK-resistant, one can conclude only that, to date, all sufficiently well characterized forms of PrP-res are both high in beta sheet and multimeric. Since conformational change and polymerization have not been convincingly separated temporally, one cannot yet determine which parameter might be most relevant in TSE pathogenesis and transmission. It must be noted that not all aggregates of PrP are PK-resistant, high in beta sheet, and associated with infectivity (23), nor are all PK-resistant, high beta sheet aggregates the same or associated with infectivity (25). Indeed, not all binding of PrP-sen to preformed PrP-res aggregates results in conversion to PrP-res, though binding appears to be a prerequisite for PrP-induced conversion $(22,24,76,77)$. These points emphasize that only a specific mode of PrP polymerization/aggregation correlates with the characteristic partial PK-resistance of PrP-res and existence of TSE infectivity. Since this is a correlation, it remains of great importance to determine directly whether the formation of PrP-res (alone) generates new TSE infectivity. Faced with these variables and complexities, it is tempting to search for a grand unifying pathological mechanism that would account for all possible disease states related to perturbations and accidents of PrP conformation, aggregation state and sequence. However, the mechanisms and attributes of PrP that might account for TSE transmissibility may differ somewhat from those accounting for pathogenesis. Furthermore, the apparently spontaneous conversion of mutant PrP-sen molecules to PrP-res in familial TSE diseases may differ mechanistically and cell biologically from the PrP-res induced conversion of wild type PrP-sen in TSEs of infectious origin.

\section{Further Reading}

This review, after minor updating, has been reprinted (with permission) from:

Harris, D.A. Prions: Molecular and Cellular Biology. Horizon Scientific Press, Wymondham, UK 


\section{References}

1. Bessen, R.A. and Marsh, R.F. 1992. Biochemical and physical properties of the prion protein from two strains of transmissible mink encephalopathy agent. J. Virol. 66: 2096-2101.

2. Bessen, R.A. and Marsh, R.F. 1994. Distinct PrP properties suggest the molecular basis of strain variation in transmissible mink encephalopathy. J. Virol. 68: 7859-7868

3. Caughey, B.W., Dong, A., Bhat, K.S., Ernst, D., Hayes, S.F., and Caughey, W.S. 1991. Secondary structure analysis of the scrapieassociated protein PrP 27-30 in water by infrared spectroscopy. Biochemistry. 30: 7672-7680.

4. Pan, K.-M., Baldwin, M., Nguyen, J., Gasset, M., Serban, A., Groth, D., Mehlhorn, I., Huang, Z., Fletterick, R.J., Cohen, F.E., and Prusiner, S.B. 1993. Conversion of alpha-helices into beta-sheets features in the formation of the scrapie prion protein. Proc. Natl. Acad. Sci. USA. 90: 10962-10966.

5. Safar, J., Roller, P.P., Gajdusek, D.C., and Gibbs, C.J., Jr. 1993 Conformational transitions, dissociation, and unfolding of scrapie amyloid (prion) protein. J. Biol. Chem. 268: 20276-20284.

6. Inouye, H. and Kirschner, D.A. 1997. X-ray diffraction analysis of scrapie prion: Intermediate and folded structures in a peptide containing two putative alpha helices. J. Mol. Biol. 268: 375-389.

7. Caughey, B. and Chesebro, B. 1997. Prion protein and the transmissible spongiform encephalopathies. Trends in Cell Biology. 7: 56-62.

8. Weissmann, C. 1996. Molecular biology of transmissible spongiform encephalopathies. FEBS Lett. 389: 3-11.

9. Prusiner, S.B. 1996. Prions.In: Fields Virology. B.N. Fields D.M. Knipe and P.M. Howley, eds. Lippincott-Raven Publishers. Philadelphia. pp. 2901-2950.

10. Horwich, A.L. and Weissman, J.S. 1997. Deadly conformations- protein misfolding in prion disease. Cell. 89: 499-510.

11. Griffith, J.S. 1967. Self-replication and scrapie. Nature. 215: 10431044.

12. Prusiner, S.B. 1982. Novel proteinaceous infectious particles cause scrapie. Science. 216: 136-144

13. Gadjusek, D C 1988. Transmissible and nontransmissible amyloidoses: Autocatalytic post-translational conversion of host precursor proteins to beta-pleated configurations. J. Neuroimmunol. 20: $95-110$.

14. Bolton, D.C. and Bendheim, P.E. 1988. A modified host protein mode of scrapie.In: Novel Infectious Agents and the Central Nervous System. G. Bock and J. Marsh, eds. John Wiley \& Sons. Chichester. pp. 164 181.

15. Jarrett, J.T. and Lansbury, P.T., Jr. 1993. Seeding "One-Dimensional Crystallization" of Amyloid: A Pathogenic Mechanism in Alzheimer's Disease and Scrapie? Cell. 73: 1055-1058

16. Kocisko, D.A., Come, J.H., Priola, S.A., Chesebro, B., Raymond, G.J., Lansbury, P.T., and Caughey, B. 1994. Cell-free formation of proteaseresistant prion protein. Nature. 370: 471-474

17. Kocisko, D.A., Lansbury, P.T., Jr., and Caughey, B. 1996. Partia unfolding and refolding of scrapie-associated prion protein: Evidence for a critical 16-kDa C-terminal domain. Biochemistry. 35: 1343413442

18. Caughey, B., Raymond, G.J., Kocisko, D.A., and Lansbury, P.T., Jr. 1997. Scrapie infectivity correlates with converting activity, protease resistance, and aggregation of scrapie-associated prion protein in guanidine denaturation studies. J. Virol. 71: 4107-4110.

19. Kaneko, K., Peretz, D., Pan, K., Blockberger, T.C., Wille, H., Gabizon, R., Griffith, O.H., Cohen, F.E., Baldwin, M.A., and Prusiner, S.B. 1995 Prion protein (PrP) synthetic peptides induce cellular PrP to acquire properties of the scrapie isoform. Proc. Natl. Acad. Sci. 92: 1116011164

20. Bossers, A., Belt, P.B.G.M., Raymond, G.J., Caughey, B., de Vries, R., and Smits, M.A. 1997. Scrapie susceptibility-linked polymorphisms modulate the in vitro conversion of sheep prion protein to proteaseresistant forms. Proc. Natl. Acad. Sci. USA. 94: 4931-4936.

21. Raymond, G.J., Hope, J., Kocisko, D.A. et al. 1997. Molecula assessment of the transmissibilities of BSE and scrapie to humans. Nature. 388: 285-288.

22. DebBurman, S.K., Raymond, G.J., Caughey, B., and Lindquist, S 1997. Chaperone-supervised conversion of prion protein to its protease-resistant form. Proc. Natl. Acad. Sci. USA. 94: 13938-13943.

23. Priola, S.A., Caughey, B., Wehrly, K., and Chesebro, B. 1995. A 60 kDa prion protein $(\mathrm{PrP})$ with properties of both the normal and scrapieassociated forms of PrP. J. Biol. Chem. 270: 3299-3305.

24. Bessen, R.A., Raymond, G.J., and Caughey, B. 1997. In situ formation of protease-resistant prion protein in transmissible spongiform encephalopathy-infected brain slices. J. Biol. Chem. 272: 15227 15231.

25. Riesner, D., Kellings, K., Post, K., Wille, H., Serban, H., Groth, D. Baldwin, M.A and Prusiner, S.B. 1996. Disruption of prion rods generates 10-nm spherical particles having high alpha-helical content and lacking scrapie infectivity. J. Virol. 70: 1714-1722.

26. Bessen, R.A., Kocisko, D.A., Raymond, G.J., Nandan, S., Lansbury, P.T., Jr., and Caughey, B. 1995. Nongenetic propagation of strainspecific phenotypes of scrapie prion protein. Nature. 375: 698-700.

27. Kaneko, K., Wille, H., Mehlhorn, I., Zhang, H., Ball, H., Cohen, F.E. Baldwin, M.A., and Prusiner, S.B. 1997. Molecular properties of complexes formed between the prion protein and synthetic peptides. J. Mol. Biol. 270: 574-586.

28. Kocisko, D.A., Priola, S.A., Raymond, G.J., Chesebro, B., Lansbury, P.T., Jr., and Caughey, B. 1995. Species specificity in the cell-free conversion of prion protein to protease-resistant forms: a model for the scrapie species barrier. Proc. Natl. Acad. Sci. USA. 92: 39233927.

29. Scott, M.R., Kohler, R., Foster, D., and Prusiner, S.B. 1992. Chimeric prion protein expression in cultured cells and transgenic mice. Protein Sci. 1: 986-997.

30. Priola, S.A., Caughey, B., Race, R.E., and Chesebro, B. 1994. Heterologous PrP molecules interfere with accumulation of proteaseresistant PrP in scrapie-infected murine neuroblastoma cells. J. Virol. 68: 4873-4878.

31. Priola, S.A. and Chesebro, B. 1995. A single hamster amino acid blocks conversion to protease-resistant $\mathrm{PrP}$ in scrapie-infected mouse neuroblastoma cells. J. Virol. 69: 7754-7758.

32. Come, J.H., Fraser, P.E., and Lansbury, P.T., Jr. 1993. A kinetic model for amyloid formation in the prion diseases: Importance of seeding. Proc. Natl. Acad. Sci. USA. 90: 5959-5963.

33. Come, J.H. and Lansbury, P.T., Jr. 1994. Predisposition of prion protein homozygotes to Creutzfeldt-Jakob disease can be explained by a nucleation-dependent polymerization mechanism. J. Am. Chem. Soc. 116: 4109-4110.

34. Nguyen, J., Baldwin, M.A., Cohen, F.E., and Prusiner, S.B. 1995. Prion protein peptides induce alpha helix to beta sheet conformational transitions. Biochemistry. 34: 4186-4192.

35. Bruce, M.E. 1996. Strain typing studies of scrapie and BSE.In: Methods in Molecular Medicine: Prion Diseases. H. Baker and R.M. Ridley, eds. Humana Press. Totowa. pp. 223-236.

36. Kascsak, R.J., Rubenstein, R., Merz, P.A., Carp, R.I., Robakis, N.K, Wisniewski, H.M., and Diringer, H. 1986. Immunological comparison of scrapie-associated fibrils isolated from animals infected with four different scrapie strains. J. Virol. 59: 676-683.

37. Monari, L., Chen, S.G., Brown, P. et al. 1994. Fatal familial insomnia and familial Creutzfeldt-Jakob disease: Different prion proteins determined by a DNA polymorphism. Proc. Natl. Acad. Sci. U. S. A. 91: 2939-2842.

38. Parchi, P., Castellani, R., Capellari, S., Ghetti, B., Young, K., Chen, S.G., Farlow, M., Dickson, D.W., Sima, A.A.F., Trojanowski, J.Q. Petersen, R.B., and Gambetti, P. 1996. Molecular basis of phenotypic variability in sporadic Creutzfeldt-Jakob disease. Ann. Neurol. 39: 767778

39. Telling, G.C., Parchi, P., DeArmond, S.J., Cortelli, P., Montagna, P., Gabizon, R., Mastrianni, J., Lugaresi, E., Gambetti, P., and Prusiner S.B. 1996. Evidence for the conformation of the pathologic isoform of the prion protein enciphering and propagating prion diversity. Science. 274: 2079-2082.

40. Merz, P.A., Somerville, R.A., Wisniewski, H.M., and Iqbal, K. 1981 Abnormal fibrils from scrapie-infected brain. Acta Neuropathol. 54: 63-74.

41. Diringer, H., Gelderblom, H., Hilmert, H., Ozel, M., Edelbluth, C., and Kimberlin, R.H. 1983. Scrapie infectivity, fibrils and low molecular weight protein. Nature. 306: 476-478.

42. Prusiner, S.B., McKinley, M.P., Bowman, K.A., Bendheim, P.E., Bolton, D.C., Groth, D.F., and Glenner, G.G. 1983. Scrapie prions aggregate to form amyloid-like birefringent rods. Cell. 35: 349-358.

43. Glenner, G.G. 1980. Amyloid deposits and amyloidosis: The betafibrillosa (second of two parts). N. Engl. J. Med. 302: 1333-1343.

44. Lansbury, P.T., Jr. and Caughey, B. 1995. The chemistry of scrapie infection: Implications of the 'ice 9' metaphor. Chem. \& Biol. 2: 1-5.

45. Gasset, M., Baldwin, M.A., Lloyd, D.H., Gabriel, J., Holtzman, D.M. Cohen, F., Fletterick, R., and Prusiner, S.B. 1992. Predicted a-helical regions of the prion protein when synthesized as peptides form amyloid. Proc. Natl. Acad. Sci. USA. 89: 10940-10944.

46. Goldfarb, L.G., Brown, P., Haltia, M., Ghiso, J., Frangione, B., and Gajdusek, D.C. 1993. Synthetic peptides corresponding to different 
mutated regions of the amyloid gene in familial Creutzfeld-Jakob disease show enhanced in vitro formation of morphologically different amyloid fibrils. Proc. Natl. Acad. Sci. USA. 90: 4451-4454.

47. Forloni, G., Angeretti, N., Chiesa, R., Monzani, E., Salmona, M., Bugiani, O., and Tagliavini, F. 1993. Neurotoxicity of a prion protein fragment. Nature. 362: 543-546.

48. Selvaggini, C., DeGioia, L., Cantu, L., Ghibaudi, E., Diomede, L., Passerini, F., Forloni, G., Bugiani, O., Tagliavini, F., and Salmona, M. 1993. Molecular characteristics of a protease-resistant, amyloidogenic and neurotoxic peptide homologous to residues 106-126 of the prion protein. Biochem. Biophys. Res. Commun. 194: 1380-1386.

49. Caughey, B., Kocisko, D.A., Raymond, G.J., and Lansbury, P.T. 1995. Aggregates of scrapie associated prion protein induce the cell-free conversion of protease-sensitive prion protein to the protease-resistant state. Chem. \& Biol. 2: 807-817.

50. Meyer, R.K., McKinley, M.P., Bowman, K.A., Braunfeld, M.B., Barry, R.A., and Prusiner, S.B. 1986. Separation and properties of cellular and scrapie prion protein. Proc. Natl. Acad. Sci. USA. 83: 2310-2314.

51. Prusiner, S.B. 1991. Molecular biology of prion diseases. Science. 252: 1515-1522.

52. Wille, H., Zhang, G., Baldwin, M.A., Cohen, F.E., and Prusiner, S.B. 1996. Separation of scrapie prion infectivity from PrP amyloid polymers. J. Mol. Biol. 259: 608-621.

53. Safar, J., Wang, W., Padgett, M.P., Ceroni, M., Piccardo, P., Zopf, D., Gajdusek, D.C., and Gibbs, C.J., Jr. 1990. Molecular mass, biochemical composition, and physicochemical behavior of the infectious form of the scrapie precursor protein monomer. Proc. Natl. Acad. Sci. USA. 87: 6373-6377.

54. Brown, P., Liberski, P., Wolff, A., and Gajdusek, D.C. 1990. Conservation of infectivity in purified fibrillary extracts of scrapieinfected hamster brain after sequential enzymatic digestion or polyacrylamide gel electrophoresis. Proc. Natl. Acad. Sci. USA. 87: 7240-7244

55. Rubenstein, R., Carp, R.I., Ju, W., Scalici, C., Papini, M.C., Rubenstein, A., and Kascsak, R.J. 1994. Concentration and distribution of infectivity and $\mathrm{PrP}^{\mathrm{Sc}}$ following partial denaturation of a mouse-adapted and $\mathrm{a}$ hamster-adapted scrapie strain. Arch. Virol. 139: 301-311.

56. Hope, J. 1994. The nature of the scrapie agent: The evolution of the virino. Ann. N. Y. Acad. Sci. 724: 282-289.

57. Janson, J., Soeller, W.C., Roche, P.C., Nelson, R.T., Torchia, A.J., Kreutter, D.K., and Butler, P.C. 1996. Spontaneous diabetes mellitus in transgenic mice expressing human islet amyloid polypeptide. Proc. Natl. Acad. Sci. USA. 93: 7283-7288.

58. Harper, J.D., Wong, S.S., Lieber, C.M., and Lansbury, P.T., Jr. 1997. Observation of metastable A-beta amyloid protofibrils by atomic force microscopy. Chem. \& Biol. 4: 119-125.

59. Lundberg, K.M., Stenland, C.J., Cohen, F.E., Prusiner, S.B., and Millhauser, G.L. 1997. Kinetics and mechanism of amyloid formation by the prion protein $\mathrm{H} 1$ peptide as determined by time-dependent ESR. Chem. \& Biol. 4: 345-355.

60. Jeffrey, M., Goodsir, C.M., Bruce, M.E., McBride, P.A., Scott, J.R., and Halliday, W.G. 1992. Infection specific prion protein (PrP) accumulates on neuronal plasmalemma in scrapie infected mice. Neurosci. Lett. 147: 106-109.

61. Jeffrey, M., Goodsir, C.M., Bruce, M.E., McBride, P.A., and Farquhar, C. 1994. Morphogenesis of amyloid plaques in $87 \mathrm{~V}$ murine scrapie. Neuropathology and Applied Neuropathology. 20: 535-542.

62. Jeffrey, M., Goodsir, C.M., Bruce, M.E., McBride, P.A., Fowler, N. and Scott, J.R. 1994. Murine scrapie-infected neurons in vivo release excess prion protein into the extracellular space. Neurosci. Lett. 174: 39-42.

63. Caughey, B. and Race, R.E. 1992. Potent inhibition of scrapieassociated PrP accumulation by Congo red. J. Neurochem. 59: 768771.

64. Caughey, B. and Raymond, G.J. 1993. Sulfated polyanion inhibition of scrapie-associated PrP accumulation in cultured cells. J. Virol. 67: 643-650.

65. Caughey, B., Ernst, D., and Race, R.E. 1993. Congo red inhibition of scrapie agent replication. J. Virol. 67: 6270-6272

66. Caughey, B., Brown, K., Raymond, G.J., Katzenstien, G.E., and Thresher, W. 1994. Binding of the protease-sensitive form of $\operatorname{PrP}$ (prion protein) to sulfated glycosaminoglycan and Congo red. J. Virol. 68: 2135-2141.

67. Liautard, J.-P. 1993. Prions and molecular chaperones. Arch. Virol. 7: 227-243.

68. Tatzelt, J., Zuo, J., Voellmy, R., Scott, M., Hartl, U., Prusiner, S.B. and Welch, W.J. 1997. Scrapie prions selectively modify the stress response in neuroblastoma cells. Proc. Natl. Acad. Sci. USA. 92: 2944-
2948.

69. Doh-ura, K., Perryman, S., Race, R., and Chesebro, B. 1995. Identification of differentially expressed genes in scrapie-infected mouse neuroblastoma cells. Microbial Pathogenesis. 18: 1-9.

70. Edenhofer, F., Rieger, R., Famulok, M., Wendler, W., Weiss, S., and Winnacker, E.-L. 1996. Prion protein PrPc interacts with molecular chaperones of the Hsp60 family. J. Virol. 70: 4724-4728.

71. Chernoff, Y.O., Lindquist, S.L., Ono, B., Inge-Vechtomov, S.G., and Liebman, S.W. 1995. Role of the chaperone protein Hsp104 in propagation of the yeast prion-like factor [psi+]. Science. 268: 880884.

72. Westaway, D., DeArmond, S.J., Cayetano-Canlas, J., Groth, D., Foster, D., Yang, S., Torchia, M., Carlson, G.A., and Prusiner, S.B. 1994. Degeneration of skeletal muscle, peripheral nerves, and the central nervous system in transgenic mice overexpressing wild-type prion proteins. Cell. 76: 117-129.

73. Bruce, M.E., Will, R.G., Ironside, J.W. et al. 1997. Transmissions to mice indicate that 'new variant' CJD is caused by the BSE agent. Nature. 389: 498-501.

74. Hill, A.F., Desbruslais, M., Joiner, S., Sidle, K.C.L., Gowland, I., Collinge, J., Doey, L.J., and Lantos, P. 1997. The same prion strain causes VCJD and BSE. Nature. 389: 448-450.

75. Harris, D.A. 1999. Prions: Molecular and Cellular Biology. Horizon Scientific Press, Wymondham, UK

76. Horiuchi, M., Chabry, J., and Caughey, B. 1999. Specific binding of normal prion protein to the scrapie form via a localized domain initiates its conversion to the protease-resistant state. EMBO J. 18:3193-3203.

77. Horiuchi, M., Priola, S.A., Chabry, J., and Caughey, B. 2000. Interactions between heterologous forms of prion protein: Binding, inhibition of conversion, and species barriers. Proc. Natl. Acad. Sci. U.S.A. 97: 5836-5841.

78. Bossers, A., de Vries, R., and Smits, M.A. 2000. Susceptibility of sheep for scrapie as assessed by in vitro conversion of nine naturally occurring variants of PrP. J. Virol. 74: 1407-1414.

79. Caughey, B., Raymond, G.J., and Bessen, R.A. 1998. Straindependent differences in beta-sheet conformations of abnormal prion protein. J. Biol. Chem. 273: 32230-32235.

80. Safar, J., Wille, H., Itri, V., Groth, D., Serban, H., Torchia, M., Cohen, F.E., and Prusiner, S.B. 1998. Eight prion strains have $\operatorname{PrP}(\mathrm{Sc})$ molecules with different conformations. Nat. Med. 4: 1157-1165.

81. Rubenstein, R., Gray, P.C., Wehlburg, C.M., Wagner, J.S., and Tisone, G.C. 1998. Detection and discrimination of PrPSc by multi-spectral ultraviolet fluorescence. Biochem. Biophys. Res. Commun. 246: 100106. 


\section{INFORMATION FOR CONTRIBUTORS}

\begin{abstract}
Aims and Scope
Current Issues in Molecular Biology (CIMB) publishes high quality review papers, in English, in all areas of molecular biology, including plant and animal systems, human genetics, molecular microbiology, virology, and biotechnology. In addition CIMB publishes articles describing innovative technology of general interest to molecular biologists.
\end{abstract}

\section{Editorial Policy}

A paper is considered for publication on the understanding that:

- it is approved by all named authors

- the authors transfer copyright of the paper to the publisher

- it has not been previously published and does not contain tables or figures that have been published elsewhere

- it will not be published elsewhere in the same form in any language without the permission of the publisher

\section{Submission Procedure}

Manuscriptions to be considered for publication in CIMB should be sent to: The Editor, CIMB, 32 Hewitts Lane, Wymondham, Norfolk, NR18 0JA, U.K. Three copies of the manuscript including all figures and tables must be submitted. To aid rapid review it is requested that the corresponding author provides telephone and fax numbers, and an e-mail address. Once a paper is accepted for publication the corresponding author must supply a copy of the manuscript on disk, either Macintosh or IBM-PC compatible, preferably Wordperfect or Microsoft Word but other forms may also be acceptable, in addition to a final hard copy.

\section{Format and Style of Articles}

English: Manuscripts must be in perfect English. If English is not your first language please have your work checked by a colleague.

Length: Each article can be up to 5000 words plus four figures or tables.

Abstract: Each paper should include an abstract. Abstracts should be 100-200 words.

Running title: No more than 50 characters.

Headings: Headings should be at the left margin, lower case (with initial capitals) and in bold type. Sub-headings should be at the left margin, lower case (with initial capitals) and in italics.

Units: Concentration to be given in $\mathrm{g} / \mathrm{l}$, etc., or molarity, M. Use the format $\mu \mathrm{g} / \mathrm{ml}$ not $\mu \mathrm{g} \mathrm{ml}-1$. Note $\mathrm{ml}$ not $\mathrm{mL}$.

Figures: Figures should if possible be supplied on disk in PICT or TIFF format; each figure should be in a separate file. Please note: The file containing the text of your article should contain text only, do NOT integrate figures into your text files. Please also supply one copy of professional quality "cameraready" artwork. A figure legend should be supplied. Colour figures can only be accepted if the cost of reproduction is borne by the authors.

Tables: Number by Arabic numerals in order of mention in the text.

References: The reference section should include only papers that are published or in press. Unpublished or submitted work should be cited in the text, as follows (J. R. Martins, unpublished). It is the responsibility of the authors to obtain permission for personal communications and a letter of permission should be enclosed with the manuscript. References should be cited in the text in parentheses in order of date, as follows (Sambrook et al., 1989; Geller, 1996). The reference list should be in alphabetical order. Note: no space between authors' initials, place initials after surname, no parentheses for date, abbreviated journal title with full stops (periods), colon after volume number, no italics or bold. Follow the style indicated:

Altschul, S.F., Gish, W., Miller, W., Myers, E.W., and Lipman, D.J. 1990. Basic local alignment search tool. J. Mol. Biol. 215: 403-410.

Brown, R.A., Lay, M., and Wittwer, C.T. 1998. Rapid cycle amplification for construction of competitive templates. In: Genetic Engineering with PCR. R.M. Horton and R.C. Tait, eds. Horizon Scientific Press, Wymondham. p. 57-70.

Sambrook, J., Fritsch, E.F., and Maniatis, T. 1989. Molecular Cloning: A Laboratory Manual. Cold Spring Harbor Laboratory Press, Cold Spring Harbor, New York.

Sanger, F., Nicklen, S., and Coulson, A.R. 1977. DNA sequencing with chain-terminating inhibitors. Proc. Natl. Acad. Sci. USA. 74: 5463-5467.

\section{Reprints}

Reprints are available for purchase at standard rates.

\section{Rapid Publication}

Our objective is to inform authors of the decision on their manuscript within four weeks of submission. Following acceptance, a paper will normally be published in the next available issue.

\section{Subscription Information}

Full details from: Caister Academic Press, 32 Hewitts Lane, Wymondham, Norfolk, NR18 0JA, U.K. Please visit our web site www.cimb.org 


\section{Further Reading}

Caister Academic Press is a leading academic publisher of advanced texts in microbiology, molecular biology and medical research. Full details of all our publications at caister.com

- MALDI-TOF Mass Spectrometry in Microbiology Edited by: M Kostrzewa, S Schubert (2016) www.caister.com/malditof

- Aspergillus and Penicillium in the Post-genomic Era Edited by: RP Vries, IB Gelber, MR Andersen (2016) www.caister.com/aspergillus2

- The Bacteriocins: Current Knowledge and Future Prospects Edited by: RL Dorit, SM Roy, MA Riley (2016)

www.caister.com/bacteriocins

- Omics in Plant Disease Resistance Edited by: V Bhadauria (2016) www.caister.com/opd

- Acidophiles: Life in Extremely Acidic Environments Edited by: R Quatrini, DB Johnson (2016) www.caister.com/acidophiles

- Climate Change and Microbial Ecology: Current Research and Future Trend

Edited by: J Marxsen (2016)

www.caister.com/climate

- Biofilms in Bioremediation: Current Research and Emerging Technologies

Edited by: G Lear (2016)

www.caister.com/biorem

- Microalgae: Current Research and Applications Edited by: MN Tsaloglou (2016) www.caister.com/microalgae

- Gas Plasma Sterilization in Microbiology: Theory, Applications, Pitfalls and New Perspectives Edited by: H Shintani, A Sakudo (2016) www.caister.com/gasplasma

- Virus Evolution: Current Research and Future Directions Edited by: SC Weaver, M Denison, M Roossinck, et al. (2016) www.caister.com/virusevol

- Arboviruses: Molecular Biology, Evolution and Control Edited by: N Vasilakis, DJ Gubler (2016) www.caister.com/arbo

- Shigella: Molecular and Cellular Biology Edited by: WD Picking, WL Picking (2016) www.caister.com/shigella

-Aquatic Biofilms: Ecology, Water Quality and Wastewater Treatment

Edited by: AM Romaní, H Guasch, MD Balaguer (2016)

www.caister.com/aquaticbiofilms

- Alphaviruses: Current Biology

Edited by: S Mahalingam, L Herrero, B Herring (2016)

www.caister.com/alpha

- Thermophilic Microorganisms

Edited by: F Li (2015)

www.caister.com/thermophile
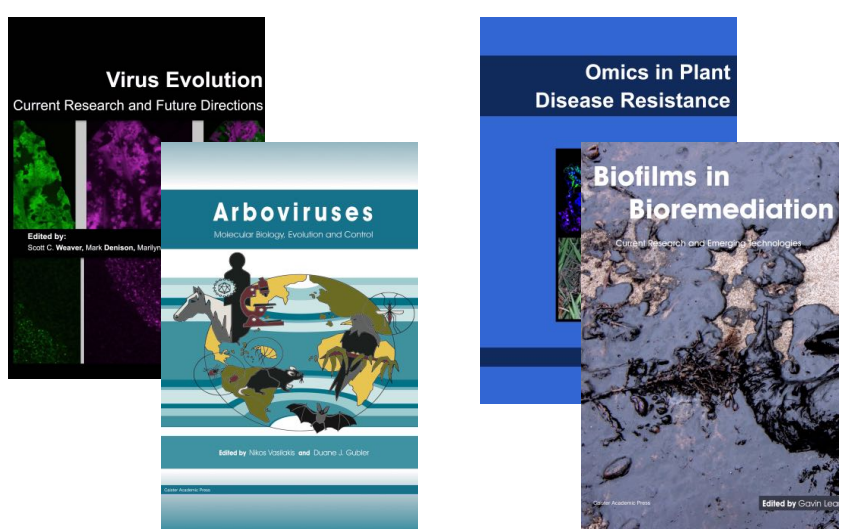
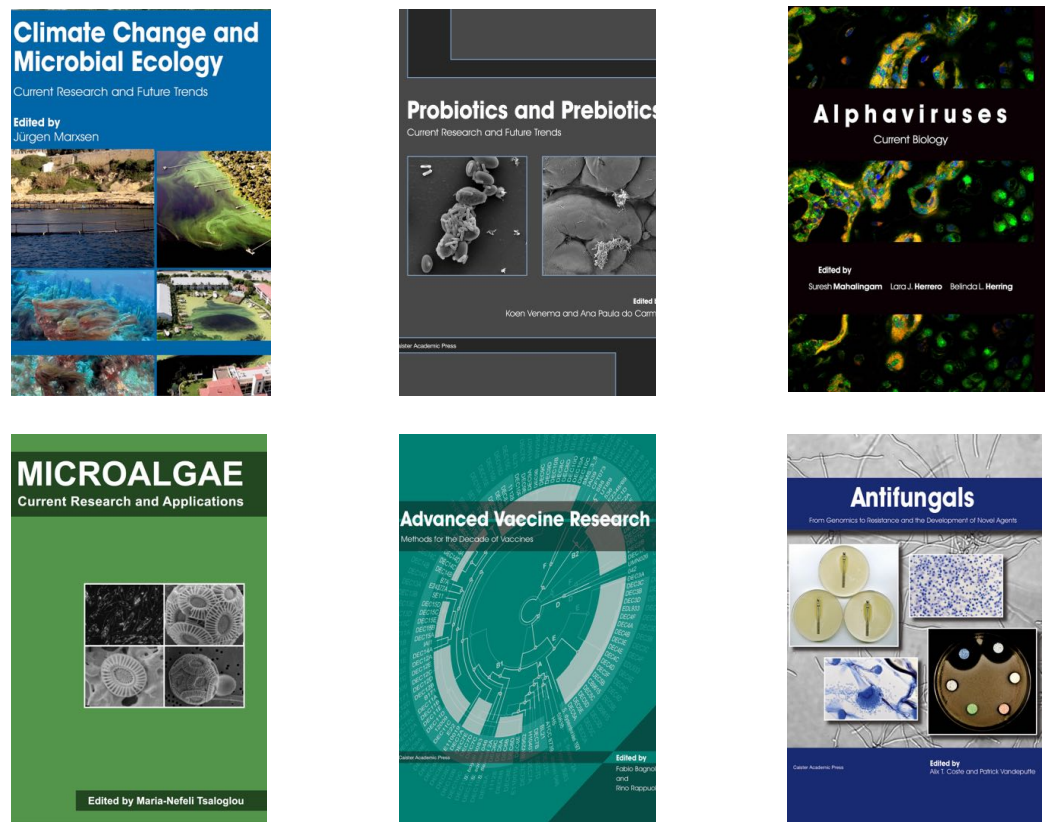

- Flow Cytometry in Microbiology: Technology and Applications Edited by: MG Wilkinson (2015) www.caister.com/flow

- Probiotics and Prebiotics: Current Research and Future Trends Edited by: K Venema, AP Carmo (2015) www.caister.com/probiotics

- Epigenetics: Current Research and Emerging Trends Edited by: BP Chadwick (2015) www.caister.com/epigenetics2015

- Corynebacterium glutamicum: From Systems Biology to Biotechnological Applications

Edited by: A Burkovski (2015)

www.caister.com/cory2

- Advanced Vaccine Research Methods for the Decade of Vaccines

Edited by: F Bagnoli, R Rappuoli (2015)

www.caister.com/vaccines

- Antifungals: From Genomics to Resistance and the Development of Novel Agents

Edited by: AT Coste, P Vandeputte (2015)

www.caister.com/antifungals

- Bacteria-Plant Interactions: Advanced Research and Future Trends Edited by: J Murillo, BA Vinatzer, RW Jackson, et al. (2015) www.caister.com/bacteria-plant

\section{- Aeromonas}

Edited by: J Graf (2015)

www.caister.com/aeromonas

- Antibiotics: Current Innovations and Future Trends

Edited by: S Sánchez, AL Demain (2015)

www.caister.com/antibiotics

- Leishmania: Current Biology and Contro Edited by: S Adak, R Datta (2015) www.caister.com/leish2

- Acanthamoeba: Biology and Pathogenesis (2nd edition) Author: NA Khan (2015)

www.caister.com/acanthamoeba2

- Microarrays: Current Technology, Innovations and Applications Edited by: Z He (2014)

www.caister.com/microarrays2

- Metagenomics of the Microbial Nitrogen Cycle: Theory, Methods and Applications

Edited by: D Marco (2014)

www.caister.com/n2 